\title{
Comparative Study of Internet Recommendation Strategies
}

\author{
Kun $L i^{1, a}$ \\ ${ }^{1}$ Haikou College of Economics Networking Academy, Haikou, Hainan, 571127 \\ ${ }^{a}$ email
}

Keywords: Recommendation Strategies, Traditional and Mobile Internet

\begin{abstract}
With the rise of the internet industry, basic necessities of life can now be settled by a phone or computer, an increasing number of transactions from the line into the line, Internet + has penetrated into every aspect of people's lives, people's quality of life has improved. At the same time, the Internet also generate huge amounts of data all the time, human history has never received such a large amount of data in a short time, these data hidden inside people's willingness to buy and all kinds of valuable public opinion towards information, if use of these data, we can produce more quality services.
\end{abstract}

\section{Introduction}

Produces vast amounts of information on the Internet every day, some produced at the time of purchase, while browsing some pages generated by these miscellaneous information hidden behind every Internet user's unique behavioral characteristics, if use of these information can be more accurate for specific users to provide better services. With the skyrocketing amount of information the Internet, to analyze such information is faced with severe challenges, how to predict the results directly recommended to the user through data mining and other related technical analyze and predict the user's behavior, and, to provide personalized information recommended service has important commercial value.

However, the growing information overload problems still plaguing Internet users. Select information of interest to the user like a needle in a haystack, but the accuracy and authenticity of the information is difficult to identify, by their own capacity constraints and cost concerns of data from the ocean, but a lot of information to the user confusion and uncertainty. In fact, the amount of information consumers want is not more or not, but for individuals to build their own interests in line with the information, in recent years, the Internet company's approach from passive information provided to active users to guess what information is needed, now, whether listening to music with the app or online shopping, there will be a "guess you like" section of personalized recommendations.

The rapid development of e-commerce to promote e-commerce website to provide better service and more specialized information, personalized service is an important research direction in the field of e-commerce, now only passive information service e-commerce platform has been unable to gain a foothold only take the initiative to attract users take the initiative to provide goods and services to the needs of users attracted users. In this owner billion users on emerging business models, driven by new science and technology to constantly develop, recommend the system plays a crucial role.

\section{Main Recommendation System Strategies}

Personalized recommendation system main algorithm complete recommendation system consists of three parts: collect user behavior information recording module analyze user preferences model analysis module, recommendation algorithm module. Among them, the recommendation algorithm module is the core part. Depending on the recommendation algorithm, recommendation system can be divided into the following categories:

Association Rules Recommendation. Follow association patterns of user behavior. As most people will buy a cigarette lighter to purchase, so you can establish a relationship between cigarettes 
and lighters, to recommend other products through this relationship. This algorithm is the most simple and direct, the user will often understood too simple, is not very intelligent algorithms, this algorithm basically been eliminated, and only a few small-scale shopping site or recommend this mode. Use history information of the user, the user computing similarity between; the use of high similarity with the target users of the evaluation of other products for users to predict the extent of the target user preferences for a particular product; to make recommendations based on user preferences of the target level. Collaborative filtering can be divided into two categories: user-based collaborative filtering and collaborative filtering based commodities. User-based collaborative filtering is the first generation of collaborative filtering technology, the principle is very simple: people in groups that buy the same products on the site that consumer should have a similar interest preferences. These systems have to find the same merchandise purchase history or the history of the user, then they have not purchased recommend each other, such as the user A bought, but the user B has not purchased recommend to $\mathrm{B}$, and vice versa. Of course, in practical recommendation application, you'll find A similar multiple users will not buy those A while others have purchased, calculating probabilities, and then sort, and finally the top-ranking product recommendations to A.

Commodity-based collaborative filtering is currently the most personalized algorithms. Because large-scale shopping site, the number of users is far greater than the number of items, based on the use of the user's personalized algorithm will cause a lot of problems. Accordingly, based on the principle of collaborative filtering technology goods: Like attracts like, the system first determines which products have the potential link between, according to the user's purchase history, the high correlation of commodities recommended to the user. For example, "Beer - diapers" seemingly wind phase and not cattle goods, the higher the frequency if both appear in the sales record, indicating the potential relevance of the stronger commodity, it can recommend to the user. Collaborative filtering algorithm can be found in the user's potential interest preferences, so its recommended new information; it is difficult to recommend a content analysis of the product; but because it is based on a user product ratings, so new users to recommend or recommend new products to users , accuracy is not high.

Recommended content based collaborative filtering is a continuation and development of technology. User and product profiles are established; users compare the similarity with the product configuration file; recommend their profile most similar products. For example, in commercial CD recommendation, based on the contents of the user's system first analyzes a high scoring bought CD common (singer, style, etc.), and then recommend the content of interest to the user a high degree of similarity to other CD. Content-based recommendation algorithm lies in access to information and information filtering. Content-based recommendation algorithm can deal with the problem of new users, new products (cold start); recommend new products and non-popular products, find hidden information; but if just two different products using the same descriptive words, these two products will not be able to distinguish; and if the system is recommended only with the user's profile highly relevant products, it is recommended that just before the user with a high degree of similarity products purchased products can not achieve the recommended diversity.

We must first create a user - dimensional product associated networks. The content features and product users as abstract nodes, the algorithm uses all the information hidden in the relationship between the user and select the product. A goal for any user, assume A Choose all products, each product has to recommend other products A abilities, not all A Choose products according to the degree of his favorite sort, the top-ranking Tell A. Recommended based network architecture is also facing problems new users of new products. And if the relationship between the user and all associated products are taken into account, can not distinguish between long-term and short-term interest points of interest, greatly reduce the recommended accuracy.

The organic combination of the above methods, the actual recommendation system is based on the most common combination of collaborative filtering and content-based.

\section{Traditional Internet and Mobile Internet Features}

Compared with traditional Internet users, mobile users often have a more clear and true, reliable 
user identification, and easily accessible from the overview level for mobile users portray example, the mobile user demographic data is usually registered by a mobile subscriber network when filling, can learn reasoning or data mining techniques to obtain (such as based on some machines, the mobile user authorization within the permitted range, you can get information based on identity information of its place of origin, purchase by the mobile subscriber forecast its revenue information through a mobile socializing web mining mobile users work/education, etc.). in addition, you can obtain additional information by mobile users in some way. for example, access to the mobile user location information or mobile user tracks via GPS (global positioning system, global positioning system), machine learning and data mining technology mobile Internet user behavior analysis to extract user attributes feature. Unlike traditional Internet access methods, mobile features of mobile users, through portability of a mobile communication network to access the Internet as well as other characteristics such that the mobile device the user can at any time, anywhere access to the Internet, so that context-aware computing in mobile Internet applications is very important. with the development of mobile communication technology, in particular 3G, B3G gradual application of technology, the mobile users to access mobile Internet speed improved, will further promote the development of mobile Internet.

\section{Similarities and Differences of Traditional Recommendation System}

Mobile Internet is the traditional recommendation system recommended extending the system in the mobile Internet field. Compared with the traditional Internet users, the mobile users, facing more complex integration, collaboration and mobile network environment to provide ubiquitous mobile information environment, only adequate, accurate extracting and predicting the mobile user in a mobile network environment after all types of mobile content preferences, in order to effectively generate a mobile recommendation. Thus, although the basic idea of a mobile recommendation system and traditional Internet recommendation systems are similar, but the important considerations environmental impact of mobile network system brings to recommend: mobile user recommendation system in a mobile network environment, mobile and strong, their mobile information needs and recommendations need of a larger context of the impact, and poor processing power of mobile devices, small screens enter the poor, the weak wireless network bandwidth and other factors make it real-time and accuracy requirements are higher, so the recommended method for traditional Internet users and can not be directly applied to a mobile recommendation.

Mobility mobile recommendation system mainly from three aspects [6] explained: wireless access user mobility, device mobility and the user's mobile device refers to a mobile user can use a mobile device at any time, anywhere access to applications. location and time context with the mobile user more closely. for example, a mobile user lunchtime in the area a, the mobile user may dine in the vicinity, we can meet the mobile user preferences to recommend the region restaurants mobile recommendation system, based on real-time context-aware recommendation is different from the traditional Internet recommended, recommended a higher real-time requirements. mobility device refers to the device easy to carry, can move with the user wireless access technologies enables mobile the access device can access the network at any time, access to the mobile network services and information content required, which makes the information push more timely and targeted, such as commodity limited-time promotion, you can put this information in a timely manner recommended to a nearby mobile users prefer the goods due to the small screen of a mobile device, the mobile user's browsing behavior differs from traditional Internet users, the results shown and described with recommended changes to accommodate the needs of mobile users and improve the mobile user experience, such as when the information display, you should reduce the mobile user slides operation window excessive sliding operation will reduce the probability that the user clicked. in view of the user, device, there is a big difference in the external environment, the mobile Internet user behavior and user behavior there is a big difference, which makes mobile recommendation system and Internet recommended system implementation relies on the data source are not the same. 


\section{Conclusion}

Personalized recommendation is to recommend the most popular apps and social networks, integrate social networks added personalized recommendation system, with the customer's friends, family rating information to recommend products. It allows customers to friends and family to do "shopping guide" them to buy higher ratings and recommend products to customers. Recommended system as an effective means to alleviate this problem, the Internet has been widely attention and application. In recent years, the recommendation system used to relieve the burden on the user to obtain information, in academia and industry attention and has been widely used, but still there are a lot issues that need in-depth and meticulous research to improve the performance of mobile recommendation. Therefore, the recommended system has important significance and broad application prospects.

\section{References}

[1] Dobre C, Xhafa F. Intelligent services for Big Data science [J]. Future GenerationComputer Systems. 2014, 37(2): 267-281.

[2] Adomavicius G, Tuzhilin A. Toward the Next Generation of Recommender Systems: A Survey of the State-of-the-art and Possible Extensions [J]. IEEE Transaction on Knowledge and Data Engineering, 2005, 17(6): 734-749.

[3] Pazzani M, Muramatsu J, Billsus D. Syskill\& Webert: identifying interesting Websites[C].Proceedings of the 13th National Conference on Artificial Intelligence and 8th Innovative Applications of Artificial Intelligence Conference. Menlo Park, 1996: 54-61.

[4] John S, David H. Analysis of Predictive Algorithms for Collaborative Filtering [J].On uncertainty in Artificial Intelligence, 1998: 25-34.

[5] Konstan. J, Miller.B, Maltz. D. Group Lens: applying collaborative filtering to use net news [M]. Communications of the ACM, 1997: 77-87. 